\title{
UV RADIATION FIELDS IN DARK CLOUDS*
}

\author{
A. P. WHITWORTH** \\ Sterrewacht, Leiden, The Netherlands
}

(Received 14 October, 1974)

\begin{abstract}
How dark is it inside a dark cloud ? If - as is currently believed - interstellar extinction at UV wavelengths is mainly due to scattering with a strongly forward throwing phase-function, the interior of a dark cloud may be much better illuminated at UV wavelengths than its measured extinction would suggest. We consider the penetration of radiation into a dark cloud against scattering and absorption by grains; and we define a new group property for interstellar grains, the exclusion optical depth $\tau_{d} . \tau_{d}$ is a measure of the ability of the grains to exclude radiation from the interior of an externally illuminated cloud. Radiation - as measured by the radiation energy density - penetrates the cloud approximately as if against pure absorption only, with effective optical depth $\tau_{d}$. Thus $\tau_{d}$ is a conceptually and numerically useful quantity when estimating the role of UV radiation in the thermal and chemical balance within a dark cloud. Computations are made of the radiation fields in $(1200,4500) \AA$, at the centres of dark clouds with measured visual extinctions. It is found that even in very dark clouds, the radiation energy density in $(1200,1800) \AA$ may be significant, due to the high grain albedo at these short wavelengths.
\end{abstract}

\section{Introduction}

Dark clouds - and in general regions of heavy obscuration - are currently the objects of intensive investigation. On the observational side, they have been found to contain a wide range of molecular, IR continuum, thermal radio continuum and radio recombination line sources. The intimate relationships between these and other observations are not yet well understood, but interpretations usually involve the assumption that dark clouds are regions of imminent or on-going star formation. In this scheme the early evolution of the dark cloud is determined by a magneto- and/or gravothermal energy imbalance, which ultimately forces some parts of the gas to form local subcondensations or protostars: the later evolution involves the contraction of these protostars to form stars, and their subsequent interaction with the remaining gas of the dark cloud. During the early evolution of the dark cloud (i.e., until the gas is heated substantially by the release of its own gravitational and nuclear energy in forming a star, or is ionized by radiation from a newly formed star in the vicinity), the physical state of the gas is observable mainly through its molecular lines. However, the interpretation of these observations, either in terms of chemical balance, or in terms of excitation conditions, is difficult. One critical uncertainty is the UV radiation field within a dark cloud. Firstly, we do not know what flux of radia-

* Paper presented at the Symposium on Solid State Astrophysics, held at the University College, Cardiff, Wales, between 9-12 July, 1974.

** As from October 1974, Department of Applied Mathematics and Astronomy, University College, Cardiff, Wales.

Astrophysics and Space Science 34 (1975) 155-173. All Rights Reserved

Copyright (C) 1975 by D. Reidel Publishing Company, Dordrecht-Holland

(C) Kluwer Academic Publishers - Provided by the NASA Astrophysics Data System 
tion is incident on the cloud. Secondly, we do not know how efficiently the flux penetrates the interior of the cloud. It is this second problem which we consider here.

On the assumption that the transfer of radiation at most wavelengths is dominated by its interaction with dust grains, we proceed in Section 2 to outline the grain parameters involved in the equation of transfer. In Section 3 we define two simple radiative transfer models; and in Section 4 we derive, in integral form, the equations of transfer for radiation being scattered and absorbed, without any (frequency coherent) re-emission. These equations are then translated to a simple finite difference form, in order to facilitate the treatment of multiple scattering (Section 5). The numerical results are presented in Section 6. It is found that radiation penetrates into the dark cloud approximately as if against pure absorption only, with effective optical depth $\tau_{d}$, which we call the exclusion optical depth. $\tau_{d}$ is a function of the orthodox grain parameters $\tau_{e}$ (extinction optical depth), $\gamma$ (grain albedo), and $g$ (mean scattering-angle cosine). In Section 7 we discuss the conceptual and numerical usefulness of the quantity $\tau_{d}$, in the context of the thermal and chemical balance within a dark cloud. Finally, we estimate the radiation fields in $(1200,4500) \AA$ at the centres of dark clouds with measured visual extinctions.

\section{Grain Properties}

The interaction between radiation and spherical homogeneous grains is described by three quantities: the absorption cross-section $\sigma_{a}$; the scattering cross-section $\sigma_{s}$; and the scattering phase-function $P_{s}(\theta) 2 \pi \sin (\theta) \mathrm{d} \theta$, which gives the probability that radiation is scattered through an angle in $(\theta, \theta+\mathrm{d} \theta)$. In astronomy, $\sigma_{a}$ and $\sigma_{s}$ are commonly replaced by the extinction cross-section $\sigma_{e}=\sigma_{a}+\sigma_{s}$, and the albedo $\gamma=$ $\sigma_{s} / \sigma_{e}$. Likewise, $P_{s}(\theta)$ is often characterized by a parameter

$$
g=\overline{\cos (\theta)}=\frac{1}{\sigma_{s}} \int_{0}^{\pi} P_{s}(\theta) 2 \pi \cos (\theta) \sin (\theta) \mathrm{d} \theta,
$$

where the integration is over all solid angles. $g$ is the mean scattering-angle cosine. (Thus a large positive $g$ implies strong forward scattering; whilst $g=0$ suggests isotropic scattering, and a large negative $g$ implies strong backward scattering.) With each cross-section is associated an efficiency $q$ (as, for example, the extinction efficiency $q_{e}=\sigma_{e} / \sigma_{g}$, where $\sigma_{g}$ is the geometric cross-section of the grain); and an optical depth $\tau$ (e.g. the extinction optical depth $\tau_{e}=N_{g} \sigma_{e}$, where $N_{g}$ is the column density of grains).

The extinction cross-section $\sigma_{e}$ measures the ability of the grain to remove radiation from a parallel beam by absorption and scattering. If we map a dark cloud by performing star counts, we only detect direct (i.e. parallel) stellar flux, and thus we obtain the extinction optical depth $\tau_{e}$ through the cloud. Similarly, if we measure 
the reddening of individual stars, we obtain the wavelength-differential of the extinction optical depth through the cloud $\Delta \tau_{e} / \Delta \lambda$.

However, $\tau_{e}$ is not in itself a reliable measure of how dark it is within the cloud. There is a growing body of evidence which indicates that a large part of the interstellar extinction at UV wavelengths derives from scattering, $0.5 \lesssim \gamma \lesssim 1.0$; and that the scattering phase-function is strongly forward throwing over a large part of the UV wavelength range, $0.25 \lesssim g \lesssim 1.0$ (see, for example, Witt and Lillie, 1973). If this is true, then diffuse stellar flux penetrates into a dark cloud much more efficiently than direct stellar flux penetrates through the cloud. Thus the interior of the cloud may be quite well illuminated. For consider the flux $F=1$ associated with a single photon incident normally on the cloud boundary. Following an absorption event, $F=0$. But if the photon is scattered through an angle $\theta$, then $F=\cos (\theta)$. Thus if $\theta$ is small $(g \rightarrow 1)$, the flux is only slightly attenuated by the scattering event. Multiple scattering will randomize the photon direction $(\bar{F}=0)$ after

$$
\mathscr{N} \sim\left(\frac{\pi}{2 \cos ^{-1}(g)}\right)^{2}
$$

scattering events (thus after one scattering if the phase-function is isotropic, $g=0$ ). However, even then the flux is not necessarily zero, since there is a probability of $\frac{1}{2}$ that the photon is still moving forward into the cloud. Nevertheless, dark clouds do appear as noticeably dark patches on the sky, even if individual stars are not resolved. Therefore we do still require that diffuse stellar flux is in some sense attenuated as it penetrates the cloud.

It should be noted that all the above grain properties $\left(\sigma_{a}, \sigma_{s}, P_{s}(\theta)\right.$, etc) depend on the wavelength of the radiation involved. This dependence has been suppressed here for the following reason. In general, the transfer of continuum radiation against grain opacity involves two interactions: scattering events, which are essentially frequency coherent; and the absorption/emission balance of the grain, which is not frequency coherent, but rather tends to degrade the photon energies. More specifically, most grains absorb a large fraction of their thermal energy from dilute optical and UV radiation fields; whilst this absorption is offset by thermal emission at much longer IR wavelengths. To a good approximation, the absorption/emission balance determines the temperature of the grain in most astronomical situations. If we accept current ideas about the constitution of grains, we find that grains cannot emit any appreciable UV radiation, because if they do, they are so hot that they will rapidly vaporize, and will not be easy to replace. Grains in dark clouds are expected to be much cooler than this, and to be emitting thermal radiation at far infrared wavelengths. Thus, in evaluating the UV radiation field inside a dark cloud, we can ignore thermal emission by the grains, and limit our considerations to the externally incident photons. These are preserved during scattering events, and are destroyed by absorption: they are never replaced in any significant numbers by thermal emission. It is for this 
reason that we need only to know the grain properties at the wavelength under consideration.

By contrast, if thermal emission were important, as it is at longer wavelengths, we should need to know the grain properties over a wide range of wavelengths. We should then have to solve a system of coupled equations describing the simultaneous transfer of radiation at all wavelengths and the thermal balance of the grains at each position in the cloud (e.g. Werner and Salpeter, 1969; Whitworth, 1972). This more comprehensive problem is in fact no more complex algebraically than the one treated here; but it immediately involves us in a detailed discussion of the wavelength dependence of the grain properties which we wish to avoid at this stage (see, for example, Wickramasinghe and Nandy, 1972). We are concerned here with the description of a purely radiative transfer phenomenon. We shall see later how this is directly relevant to the evaluation of UV radiation fields in dark clouds. In the meantime, we can meaningfully continue to suppress the wavelength dependence of the grain properties: this will serve to underline the generality of the formulism.

In addition, we note that the optical properties of a spherical grain $\left(\sigma_{a}, \sigma_{s}, P_{s}(\theta)\right.$, etc.) are functions of the grain size. In the case of a grain whose structure is anisotropic, the optical properties are also functions of the orientation of the grain with respect to the incoming photon, and of the polarization of the incoming photon. We shall assume that the distribution of grain sizes, shapes, structures, constituents and orientations in a dark cloud can be adequately represented by the optical properties of a single average spherical grain (thus $\sigma_{a}, \sigma_{s}, P_{s}(\theta)$, etc.), and we shall use these properties in our subsequent calculations. (This approximation is only strictly acceptable if any anisotropic grains are not strongly aligned, and/or if the illumination of the cloud is not very anisotropic. In reality, there may be strong grain alignment due to a frozen-in magnetic field (Carrasco et al., 1973); and the illumination may be highly anisotropic due to newly formed bright stars in the immediate vicinity.)

\section{Radiative Transfer Model}

Firstly, we model the cloud by a semi-infinite, plane-parallel slab. Positions within the cloud are measured in terms of the optical depth $\tau$, normal to the 'near' cloud boundary at $\tau=0$. The 'far' cloud boundary is at $\tau=\tau_{0}$. Outside the cloud, i.e. outside the range $0 \leqslant \tau \leqslant \tau_{0}$, the medium is completely transparent.

Secondly, for the radiation field incident on the cloud boundary, we consider two extreme cases. Case a: diffuse illumination. The radiation field at the cloud boundary is semi-isotropic. In terms of the intensity $I(\tau, \mu)$, we write

$$
I_{0}(\tau=0, \mu>0)=I_{*}=\frac{U_{*} c}{4 \pi}=I_{0}\left(\tau=\tau_{0}, \mu<0\right) .
$$

$\mu=\cos (\phi)$, and $\phi$ is the angle between the intensity and the $\tau$-axis. $U_{*}$ is to be identified with the radiation density in an unobscured region far outside the cloud. 
Case b: direct illumination. A parallel beam of radiation is incident normally on both cloud boundaries:

$$
I_{0}(\tau=0, \mu>0)=\frac{U_{*} \delta(1-|\mu|) c}{4 \pi}=I_{0}\left(\tau=\tau_{0}, \mu<0\right) .
$$

Thirdly, we approximate the scattering phase-function $P_{s}(\theta)$ by a probability $g$ that the scattered photon is scattered straight forward (which is equivalent to its not being scattered at all), and a probability $(1-g)$ that the scattered photon is scattered isotropically. $g$ is defined by Equation (1). Evidently this approximation suppresses some details of the phase-function. The phase-function of an individual grain is generally a rather complex function of $\theta$, and possibly of other angles (see, for example, Wickramasinghe, 1973). However, when we allow for a realistic distribution of grain sizes, shapes, structures, constituents and orientations in the interstellar medium, it is likely that very little remains except a tendency for the grains to scatter radiation forward or backward, more or less strongly. In the first approximation, $g$ is a convenient measure of this tendency (cf. Aannestad and Purcell, 1973). With the scattering phase-function approximated in this way, we define two new variables: the effective extinction cross-section,

$$
\sigma=\sigma_{e}(1-g \gamma)
$$

and the effective albedo,

$$
\alpha=\frac{\gamma(1-g)}{(1-g \gamma)}
$$

The radiative transfer equations are now formulated in terms of $\alpha$ and the effective extinction optical depth

$$
\tau=N_{g} \sigma
$$

\section{Equations of Transfer: Integral Formulation}

We denote by a subscript ' $i$ ' the density of radiation which has been scattered $i$ times. The penetration of radiation into the cloud is measured by the total radiation density $U_{t}(\tau)$, normalized to the density of radiation $U_{*}$ far outside the cloud as

$$
u(\tau)=\frac{U_{t}(\tau)}{U_{*}}
$$

In principle, $u(\tau)$ can be derived from the following three equations

$$
\begin{aligned}
& u_{0}(\tau)=\begin{array}{l}
\frac{1}{2}\left(E_{2}(\tau)+E_{2}\left(\tau_{0}-\tau\right)\right), \quad \text { case } \mathrm{a} ; \\
\frac{1}{2}\left(\exp (-\tau)+\exp \left(\tau-\tau_{0}\right)\right), \quad \text { case b; }
\end{array} \\
& u_{i}(\tau)=\frac{\alpha}{2} \int_{0}^{\tau_{0}} u_{i-1}\left(\tau^{\prime}\right) E_{1}\left(\left|\tau-\tau^{\prime}\right|\right) \mathrm{d} \tau^{\prime}, \quad i \geqslant 1 ;
\end{aligned}
$$




$$
u(\tau)=\sum_{i=0}^{\infty}\left(u_{i}(\tau)\right)=\frac{U_{t}(\tau)}{U_{*}}
$$

The functions $E_{1}(x), E_{2}(x)$, etc. are exponential integrals defined by

$$
E_{n}(x)=\int_{1}^{\infty} \frac{\exp (-x y)}{y^{n}} \mathrm{~d} y, \quad n \geqslant 1 .
$$

Their analytic and numerical properties are discussed in Abramowitz and Stegun (1964, p. 227f.).

\section{Equations of Transfer: Finite Difference Formulation}

The iterative routine contained in Equation (10) must be treated numerically. We divide the cloud into $2 \mathscr{N}$ plane-parallel slab elements of equal optical thickness $\delta \tau=\tau_{0} / 2 \mathscr{N}$. The $j$-th element is contained in $(\tau(j-1), \tau(j))$, where $\tau(j)=j \tau_{0} / 2 \mathscr{N}$. The normalized radiation densities $u_{i}(\tau)$ are averaged over $\tau$ within each element, at each iteration (i.e. at each scattering order); and are then treated as being uniform across the element in computing the next iteration. We denote these average values by a bar as $\bar{u}_{i}(j)$. The equations of transfer ( 9 to 11 ) reduce to the form

$$
\begin{aligned}
& \left(E_{3}(\tau(j-1))-E_{3}(\tau(j))+E_{3}(\tau(2 \mathscr{N}-j))-\right. \\
& \bar{u}_{0}(j)=\frac{\left.-E_{3}(\tau(2 \mathscr{N}+1-j))\right)}{(2 \delta \tau)}, \quad \text { case a; } \\
& (\exp (-\tau(j-1))-\exp (-\tau(j))+\exp (-\tau(2 \mathscr{N}-j))- \\
& \bar{u}_{0}(j)=\frac{-\exp (-\tau(2 \mathscr{N}+1-j)))}{(2 \delta \tau)}, \\
& \text { case } b ; \quad(13 b) \\
& \bar{u}_{i}(j)=\frac{\alpha}{2 \delta \tau} \sum_{k=1}^{2 \mathscr{N}}\left(\bar{u}_{i-1}(k) \mathrm{d}(k, j)\right) \\
& \mathrm{d}(k, j)=E_{3}(\tau(j-k-1))+E_{3}(\tau(j-k+1))- \\
& -2 E_{3}(\tau(j-k)), \quad k<j \text {; } \\
& =2 \delta \tau-1+2 E_{3}(\delta \tau), \quad k=j \text {; } \\
& =\mathrm{d}(j, k), \quad k>j \text {. } \\
& \bar{u}(j)=\sum_{i=0}^{\mathscr{M}_{c}}\left(\bar{u}_{i}(j)\right) .
\end{aligned}
$$

The numerical procedure contained in Equations (13a) through (16) divides into two parts. Firstly, we must evaluate the zero order solution (Equation (13a) or (13b)), and the elements of the coupling matrix $\mathrm{d}(j, k)$ (Equations $(15 \mathrm{a}, \mathrm{b}, \mathrm{c})$ ). The computing 
time required for this is governed by the time required to evaluate the exponential integrals with sufficient accuracy. Secondly, we enter the iterative routine described by Equation (14), and use this to generate the contribution $\bar{u}_{i}(j)$ of each scattering order to the total normalized radiation density. These contributions are then summed according to Equation (16), to obtain the total mean normalized radiation density $\bar{u}(j)$ in each element. This summation is terminated for $\mathscr{M}_{c}$ terms (i.e. $\mathscr{M}_{c}$ orders of scattering) as soon as adequate convergence is obtained. Since the iterative routine is extremely simple, it is not prohibitive to follow a large number of scatterings when this is necessary (i.e. for large $\tau_{e 0}$, and/or large $\gamma$, and/or small $g$ ).

\section{Numerical Results}

We have solved the equations of transfer in finite difference form for a grid of: (i) grain albedos, $\gamma=0.10,0.30,0.50,0.70,0.90,0.95$; (ii) mean scattering-angle cosines, $g=0.00,0.10,0.25,0.50,0.75,0.90,0.95$; and (iii) cloud sizes, as measured by the total extinction optical depth through the cloud, $\tau_{e 0}=1,2,4,8,16,32$. We have considered the two geometries defined in Section 3, namely a plane-parallel slab illuminated (a) diffusely by a semi-isotropic radiation field, and (b) directly by a parallel beam at normal incidence. Eighty optical depth elements were used for each case. The results are determined in terms of the dimensionless variable $\bar{u}(j)$, which is the mean radiation energy density in element $j$, normalized to a value unity far outside the cloud. It is not feasible or useful to present the results in full. Rather, we shall simply note and discuss the most important features.

In Table I we give values of $u_{c}$, the normalized radiation density at the cloud centre, on a grid of $\left(\gamma, g, \tau_{e 0}\right)$ values for illumination case a. The first column gives the value of $\gamma$, and the second column gives the value of $g$. The remaining six columns give values of $u_{c}$ in floating point notation. (In this and all subsequent tables, an entry of the form $1.23(-45)$ is equivalent to $1.23 \times 10^{-45}$.) Each of the last six columns is headed by the appropriate value of the total extinction optical depth $\tau_{e 0}$. Table II is the same as Table I, but for illumination case b. Evidently, if $\gamma$ and/or $g$ is large (strong scattering/forward throwing), $u_{c}$ may be substantially larger than if the extinction were due purely to absorption $(\gamma=0)$. In fact, for illumination case $b$ (Table II) and sufficiently large $\gamma, u_{c}$ may actually exceed unity at the centres of thin clouds. This is a real effect due to the anisotropic illumination in case $b$, and is not a violation of basic physical principles.

The form of $u(\tau)$ in $\left(0, \tau_{0}\right)$ has in all cases a shape which approximates to that which would obtain if the grains did not scatter, but only absorbed radiation, with an effective optical depth $\tau_{d}$ :

$$
\begin{aligned}
& u(\tau)=\frac{1}{2}\left(E_{2}\left(\tau_{d}\right)+E_{2}\left(\tau_{d 0}-\tau_{d}\right)\right), \quad \text { case } \mathrm{a} ; \\
& u(\tau)=\frac{1}{2}\left(\exp \left(-\tau_{d}\right)+\exp \left(\tau_{d}-\tau_{d 0}\right)\right), \quad \text { case } \mathrm{b} ; \\
& \tau_{d}=\tau_{e} G_{d} .
\end{aligned}
$$


TABLE I

The normalized central radiation density $u_{c}$, for various total extinction optical depths $\tau_{e 0}$, and illumination case a

\begin{tabular}{|c|c|c|c|c|c|c|c|}
\hline \multirow[t]{2}{*}{$\gamma$} & \multirow[t]{2}{*}{$g$} & \multicolumn{6}{|l|}{$u_{c}$} \\
\hline & & $\tau_{e 0}=1$ & $\tau_{e 0}=2$ & $\tau_{e 0}=4$ & $\tau_{e 0}=8$ & $\tau_{e 0}=16$ & $\tau_{e 0}=32$ \\
\hline 0.10 & 0.00 & $3.53(-1)$ & $1.66(-1)$ & $4.37(-2)$ & $3.93(-3)$ & $4.49(-5)$ & $8.98(-9)$ \\
\hline 0.10 & 0.10 & $3.53(-1)$ & $1.66(-1)$ & $4.42(-2)$ & $4.04(-3)$ & $4.77(-5)$ & $1.03(-8)$ \\
\hline 0.10 & 0.25 & $3.54(-1)$ & $1.67(-1)$ & $4.49(-2)$ & $4.20(-3)$ & $5.23(-5)$ & $1.25(-8)$ \\
\hline 0.10 & 0.50 & $3.55(-1)$ & $1.69(-1)$ & $4.62(-2)$ & $4.50(-3)$ & $6.10(-5)$ & $1.75(-8)$ \\
\hline 0.10 & 0.75 & $3.55(-1)$ & $1.71(-1)$ & $4.75(-2)$ & $4.81(-3)$ & $7.11(-5)$ & $2.46(-8)$ \\
\hline 0.10 & 0.90 & $3.56(-1)$ & $1.72(-1)$ & $4.83(-2)$ & $5.01(-3)$ & $7.80(-5)$ & $3.01(-8)$ \\
\hline 0.30 & 0.00 & $4.18(-1)$ & $2.12(-1)$ & $6.22(-2)$ & $6.46(-3)$ & $8.97(-5)$ & $2.33(-8)$ \\
\hline 0.30 & 0.10 & $4.19(-1)$ & $2.15(-1)$ & $6.41(-2)$ & $6.95(-3)$ & $1.06(-4)$ & $3.37(-8)$ \\
\hline 0.30 & 0.25 & $4.20(-1)$ & $2.18(-1)$ & $6.70(-2)$ & $7.75(-3)$ & $1.36(-4)$ & $5.87(-8)$ \\
\hline 0.30 & 0.50 & $4.23(-1)$ & $2.24(-1)$ & $7.22(-2)$ & $9.33(-3)$ & $2.09(-4)$ & $1.51(-7)$ \\
\hline 0.30 & 0.75 & $4.25(-1)$ & $2.29(-1)$ & $7.78(-2)$ & $1.13(-2)$ & $3.22(-4)$ & $3.95(-7)$ \\
\hline 0.30 & 0.90 & $4.26(-1)$ & $2.33(-1)$ & $8.14(-2)$ & $1.26(-2)$ & $4.20(-4)$ & $7.09(-7)$ \\
\hline 0.50 & 0.00 & $5.07(-1)$ & $2.86(-1)$ & $9.71(-2)$ & $1.25(-2)$ & $2.43(-4)$ & $1.08(-7)$ \\
\hline 0.50 & 0.10 & $5.09(-1)$ & $2.90(-1)$ & $1.01(-1)$ & $1.39(-2)$ & $3.09(-4)$ & $1.83(-7)$ \\
\hline 0.50 & 0.25 & $5.11(-1)$ & $2.97(-1)$ & $1.08(-1)$ & $1.63(-2)$ & $4.46(-4)$ & $4.11(-7)$ \\
\hline 0.50 & 0.50 & $5.15(-1)$ & $3.07(-1)$ & $1.20(-1)$ & $2.14(-2)$ & $8.38(-4)$ & $1.68(-6)$ \\
\hline 0.50 & 0.75 & $5.17(-1)$ & $3.17(-1)$ & $1.34(-1)$ & $2.82(-2)$ & $1.62(-3)$ & $7.32(-6)$ \\
\hline 0.50 & 0.90 & $5.18(-1)$ & $3.23(-1)$ & $1.42(-1)$ & $3.35(-2)$ & $2.43(-3)$ & $1.83(-5)$ \\
\hline 0.70 & 0.00 & $6.38(-1)$ & $4.17(-1)$ & $1.77(-1)$ & $3.23(-2)$ & $1.15(-3)$ & $1.55(-6)$ \\
\hline 0.70 & 0.10 & $6.40(-1)$ & $4.23(-1)$ & $1.85(-1)$ & $3.62(-2)$ & $1.49(-3)$ & $2.71(-6)$ \\
\hline 0.70 & 0.25 & $6.43(-1)$ & $4.32(-1)$ & $1.98(-1)$ & $4.32(-2)$ & $2.23(-3)$ & $6.52(-6)$ \\
\hline 0.70 & 0.50 & $6.46(-1)$ & $4.47(-1)$ & $2.22(-1)$ & $5.85(-2)$ & $4.55(-3)$ & $3.17(-5)$ \\
\hline 0.70 & 0.75 & $6.46(-1)$ & $4.60(-1)$ & $2.49(-1)$ & $8.02(-2)$ & $9.82(-3)$ & $1.82(-4)$ \\
\hline 0.70 & 0.90 & $6.44(-1)$ & $4.67(-1)$ & $2.65(-1)$ & $9.75(-2)$ & $1.60(-2)$ & $5.68(-4)$ \\
\hline 0.90 & 0.00 & $8.45(-1)$ & $6.99(-1)$ & $4.46(-1)$ & $1.62(-1)$ & $2.00(-2)$ & $3.07(-4)$ \\
\hline 0.90 & 0.10 & $8.46(-1)$ & $7.05(-1)$ & $4.60(-1)$ & $1.76(-1)$ & $2.41(-2)$ & $4.55(-4)$ \\
\hline 0.90 & 0.25 & $8.48(-1)$ & $7.13(-1)$ & $4.81(-1)$ & $2.00(-1)$ & $3.24(-2)$ & $8.50(-4)$ \\
\hline 0.90 & 0.50 & $8.49(-1)$ & $7.26(-1)$ & $5.19(-1)$ & $2.50(-1)$ & $5.56(-2)$ & $2.75(-3)$ \\
\hline 0.90 & 0.75 & $8.46(-1)$ & $7.35(-1)$ & $5.56(-1)$ & $3.16(-1)$ & $1.02(-1)$ & $1.12(-2)$ \\
\hline 0.90 & 0.90 & $8.39(-1)$ & $7.33(-1)$ & $5.73(-1)$ & $3.62(-1)$ & $1.53(-1)$ & $3.04(-2)$ \\
\hline 0.95 & 0.00 & $9.16(-1)$ & $8.26(-1)$ & $6.32(-1)$ & $3.23(-1)$ & $7.28(-2)$ & $3.58(-3)$ \\
\hline 0.95 & 0.10 & $9.17(-1)$ & $8.30(-1)$ & $6.44(-1)$ & $3.43(-1)$ & $8.36(-2)$ & $4.77(-3)$ \\
\hline 0.95 & 0.25 & $9.18(-1)$ & $8.36(-1)$ & $6.64(-1)$ & $3.75(-1)$ & $1.04(-1)$ & $7.57(-3)$ \\
\hline 0.95 & 0.50 & $9.19(-1)$ & $8.44(-1)$ & $6.96(-1)$ & $4.39(-1)$ & $1.55(-1)$ & $1.81(-2)$ \\
\hline 0.95 & 0.75 & $9.16(-1)$ & $8.49(-1)$ & $7.25(-1)$ & $5.14(-1)$ & $2.44(-1)$ & $5.21(-2)$ \\
\hline 0.95 & 0.90 & $9.10(-1)$ & $8.45(-1)$ & $7.35(-1)$ & $5.61(-1)$ & $3.28(-1)$ & $1.13(-1)$ \\
\hline
\end{tabular}


TABLE II

The normalized central radiation density $u_{c}$, for various total extinction optical depths $\tau_{20}$, and illumination case $b$

\begin{tabular}{|c|c|c|c|c|c|c|c|}
\hline \multirow[t]{2}{*}{$\gamma$} & \multirow[t]{2}{*}{$g$} & \multicolumn{6}{|l|}{$u_{c}$} \\
\hline & & $\tau_{e 0}=1$ & $\tau_{e 0}=2$ & $\tau_{e 0}=4$ & $\tau_{e 0}=8$ & $\tau_{e 0}=16$ & $\tau_{e 0}=32$ \\
\hline 0.10 & 0.00 & $6.51(-1)$ & $4.05(-1)$ & $1.53(-1)$ & $2.13(-2)$ & $4.05(-4)$ & $1.42(-7)$ \\
\hline 0.10 & 0.10 & $6.50(-1)$ & $4.05(-1)$ & $1.54(-1)$ & $2.19(-2)$ & $4.31(-4)$ & $1.63(-7)$ \\
\hline 0.10 & 0.25 & $6.48(-1)$ & $4.05(-1)$ & $1.56(-1)$ & $2.27(-2)$ & $4.72(-4)$ & $2.00(-7)$ \\
\hline 0.10 & 0.50 & $6.45(-1)$ & $4.06(-1)$ & $1.59(-1)$ & $2.42(-2)$ & $5.50(-4)$ & $2.82(-7)$ \\
\hline 0.10 & 0.75 & $6.41(-1)$ & $4.06(-1)$ & $1.62(-1)$ & $2.57(-2)$ & $6.41(-4)$ & $3.97(-7)$ \\
\hline 0.10 & 0.90 & $6.39(-1)$ & $4.06(-1)$ & $1.64(-1)$ & $2.67(-2)$ & $7.03(-4)$ & $4.88(-7)$ \\
\hline 0.30 & 0.00 & $7.62(-1)$ & $5.03(-1)$ & $2.04(-1)$ & $3.09(-2)$ & $6.56(-4)$ & $2.65(-7)$ \\
\hline 0.30 & 0.10 & $7.57(-1)$ & $5.03(-1)$ & $2.08(-1)$ & $3.32(-2)$ & $7.79(-4)$ & $3.91(-7)$ \\
\hline 0.30 & 0.25 & $7.50(-1)$ & $5.04(-1)$ & $2.15(-1)$ & $3.68(-2)$ & $1.01(-3)$ & $7.03(-7)$ \\
\hline 0.30 & 0.50 & $7.36(-1)$ & $5.03(-1)$ & $2.26(-1)$ & $4.36(-2)$ & $1.56(-3)$ & $1.88(-6)$ \\
\hline 0.30 & 0.75 & $7.21(-1)$ & $5.01(-1)$ & $2.36(-1)$ & $5.16(-2)$ & $2.40(-3)$ & $5.07(-6)$ \\
\hline 0.30 & 0.90 & $7.12(-1)$ & $4.99(-1)$ & $2.43(-1)$ & $5.70(-2)$ & $3.11(-3)$ & $9.21(-6)$ \\
\hline 0.50 & 0.00 & $9.14(-1)$ & $6.55(-1)$ & $2.94(-1)$ & $5.15(-2)$ & $1.35(-3)$ & $7.80(-7)$ \\
\hline 0.50 & 0.10 & $9.03(-1)$ & $6.55(-1)$ & $3.03(-1)$ & $5.70(-2)$ & $1.75(-3)$ & $1.38(-6)$ \\
\hline 0.50 & 0.25 & $8.87(-1)$ & $6.53(-1)$ & $3.17(-1)$ & $6.64(-2)$ & $2.58(-3)$ & $3.33(-6)$ \\
\hline 0.50 & 0.50 & $8.57(-1)$ & $6.45(-1)$ & $3.38(-1)$ & $8.52(-2)$ & $4.96(-3)$ & $1.50(-5)$ \\
\hline 0.50 & 0.75 & $8.21(-1)$ & $6.31(-1)$ & $3.56(-1)$ & $1.08(-1)$ & $9.56(-3)$ & $7.03(-5)$ \\
\hline 0.50 & 0.90 & $7.97(-1)$ & $6.17(-1)$ & $3.64(-1)$ & $1.24(-1)$ & $1.41(-2)$ & $1.80(-4)$ \\
\hline 0.70 & 0.00 & $1.13(0)$ & $9.20(-1)$ & $4.90(-1)$ & $1.11(-1)$ & $4.62(-3)$ & $6.72(-6)$ \\
\hline 0.70 & 0.10 & $1.11(0)$ & $9.16(-1)$ & $5.06(-1)$ & $1.24(-1)$ & $6.13(-3)$ & $1.23(-5)$ \\
\hline 0.70 & 0.25 & $1.08(0)$ & $9.05(-1)$ & $5.28(-1)$ & $1.47(-1)$ & $9.49(-3)$ & $3.23(-5)$ \\
\hline 0.70 & 0.50 & $1.02(0)$ & $8.74(-1)$ & $5.58(-1)$ & $1.94(-1)$ & $2.02(-2)$ & $1.83(-4)$ \\
\hline 0.70 & 0.75 & $9.52(-1)$ & $8.22(-1)$ & $5.70(-1)$ & $2.50(-1)$ & $4.35(-2)$ & $1.19(-3)$ \\
\hline 0.70 & 0.90 & $9.00(-1)$ & $7.78(-1)$ & $5.63(-1)$ & $2.83(-1)$ & $6.83(-2)$ & $3.80(-3)$ \\
\hline 0.90 & 0.00 & $1.48(0)$ & $1.48(0)$ & $1.12(0)$ & $4.51(-1)$ & $5.78(-2)$ & $8.91(-4)$ \\
\hline 0.90 & 0.10 & $1.45(0)$ & $1.46(0)$ & $1.13(0)$ & $4.90(-1)$ & $7.06(-2)$ & $1.34(-3)$ \\
\hline 0.90 & 0.25 & $1.39(0)$ & $1.41(0)$ & $1.15(0)$ & $5.54(-1)$ & $9.69(-2)$ & $2.59(-3)$ \\
\hline 0.90 & 0.50 & $1.28(0)$ & $1.31(0)$ & $1.15(0)$ & $6.70(-1)$ & $1.71(-1)$ & $9.11(-3)$ \\
\hline 0.90 & 0.75 & $1.15(0)$ & $1.16(0)$ & $1.07(0)$ & $7.62(-1)$ & $3.10(-1)$ & $4.15(-2)$ \\
\hline 0.90 & 0.90 & $1.04(0)$ & $1.03(0)$ & $9.51(-1)$ & $7.52(-1)$ & $4.17(-1)$ & $1.12(-1)$ \\
\hline 0.95 & 0.00 & $1.60(0)$ & $1.73(0)$ & $1.54(0)$ & $8.52(-1)$ & $1.96(-1)$ & $9.62(-3)$ \\
\hline 0.95 & 0.10 & $1.56(0)$ & $1.70(0)$ & $1.55(0)$ & $9.02(-1)$ & $2.26(-1)$ & $1.29(-2)$ \\
\hline 0.95 & 0.25 & $1.49(0)$ & $1.63(0)$ & $1.54(0)$ & $9.81(-1)$ & $2.85(-1)$ & $2.08(-2)$ \\
\hline 0.95 & 0.50 & $1.37(0)$ & $1.50(0)$ & $1.49(0)$ & $1.11(0)$ & $4.31(-1)$ & $5.19(-2)$ \\
\hline 0.95 & 0.75 & $1.21(0)$ & $1.29(0)$ & $1.33(0)$ & $1.15(0)$ & $6.57(-1)$ & $1.60(-1)$ \\
\hline 0.95 & 0.90 & $1.09(0)$ & $1.12(0)$ & $1.13(0)$ & $1.04(0)$ & $7.67(-1)$ & $3.38(-1)$ \\
\hline
\end{tabular}


TABLE III

Values of $G_{d}=\tau_{d} / \tau_{e}$, for various total extinction optical depths $\tau_{e 0}$, and illumination case a

\begin{tabular}{|c|c|c|c|c|c|c|c|}
\hline \multirow[t]{2}{*}{$\gamma$} & \multirow[t]{2}{*}{$g$} & \multicolumn{6}{|l|}{$G_{d}$} \\
\hline & & $\tau_{e 0}=1$ & $\tau_{e 0}=2$ & $\tau_{e 0}=4$ & $\tau_{e 0}=8$ & $\tau_{e 0}=16$ & $\tau_{e 0}=32$ \\
\hline 0.10 & 0.00 & 0.9110 & 0.9263 & 0.9420 & 0.9566 & 0.9692 & 0.9793 \\
\hline 0.10 & 0.10 & 0.9099 & 0.9236 & 0.9378 & 0.9510 & 0.9623 & 0.9714 \\
\hline 0.10 & 0.25 & 0.9081 & 0.9196 & 0.9315 & 0.9425 & 0.9520 & 0.9596 \\
\hline 0.10 & 0.50 & 0.9054 & 0.9130 & 0.9210 & 0.9283 & 0.9347 & 0.9398 \\
\hline 0.10 & 0.75 & 0.9026 & 0.9065 & 0.9105 & 0.9142 & 0.9173 & 0.9199 \\
\hline 0.10 & 0.90 & 0.9010 & 0.9026 & 0.9042 & 0.9057 & 0.9070 & 0.9080 \\
\hline 0.30 & 0.00 & 0.7228 & 0.7642 & 0.8094 & 0.8528 & 0.8913 & 0.9230 \\
\hline 0.30 & 0.10 & 0.7200 & 0.7575 & 0.7985 & 0.8377 & 0.8725 & 0.9013 \\
\hline 0.30 & 0.25 & 0.7161 & 0.7476 & 0.7820 & 0.8150 & 0.8443 & 0.8685 \\
\hline 0.30 & 0.50 & 0.7100 & 0.7313 & 0.7546 & 0.7770 & 0.7968 & 0.8132 \\
\hline 0.30 & 0.75 & 0.7044 & 0.7154 & 0.7272 & 0.7386 & 0.7487 & 0.7570 \\
\hline 0.30 & 0.90 & 0.7017 & 0.7060 & 0.7109 & 0.7155 & 0.7195 & 0.7229 \\
\hline 0.50 & 0.00 & 0.5199 & 0.5783 & 0.6476 & 0.7169 & 0.7799 & 0.8329 \\
\hline 0.50 & 0.10 & 0.5166 & 0.5697 & 0.6327 & 0.6958 & 0.7534 & 0.8020 \\
\hline 0.50 & 0.25 & 0.5122 & 0.5569 & 0.6103 & 0.6639 & 0.7129 & 0.7547 \\
\hline 0.50 & 0.50 & 0.5057 & 0.5364 & 0.5730 & 0.6100 & 0.6439 & 0.6729 \\
\hline 0.50 & 0.75 & 0.5014 & 0.5172 & 0.5361 & 0.5553 & 0.5728 & 0.5879 \\
\hline 0.50 & 0.90 & 0.5000 & 0.5066 & 0.5143 & 0.5222 & 0.5293 & 0.5355 \\
\hline 0.70 & 0.00 & 0.3038 & 0.3622 & 0.4420 & 0.5287 & 0.6096 & 0.6775 \\
\hline 0.70 & 0.10 & 0.3012 & 0.3543 & 0.4271 & 0.5067 & 0.5816 & 0.6452 \\
\hline 0.70 & 0.25 & 0.2977 & 0.3428 & 0.4047 & 0.4733 & 0.5383 & 0.5946 \\
\hline 0.70 & 0.50 & 0.2936 & 0.3248 & 0.3679 & 0.4163 & 0.4629 & 0.5042 \\
\hline 0.70 & 0.75 & 0.2930 & 0.3096 & 0.3323 & 0.3581 & 0.3833 & 0.4059 \\
\hline 0.70 & 0.90 & 0.2957 & 0.3028 & 0.3123 & 0.3231 & 0.3337 & 0.3432 \\
\hline 0.90 & 0.00 & 0.0865 & 0.1135 & 0.1632 & 0.2353 & 0.3116 & 0.3770 \\
\hline 0.90 & 0.10 & 0.0855 & 0.1100 & 0.1552 & 0.2215 & 0.2931 & 0.3554 \\
\hline 0.90 & 0.25 & 0.0844 & 0.1051 & 0.1434 & 0.2006 & 0.2642 & 0.3212 \\
\hline 0.90 & 0.50 & 0.0835 & 0.0980 & 0.1245 & 0.1651 & 0.2129 & 0.2580 \\
\hline 0.90 & 0.75 & 0.0855 & 0.0935 & 0.1078 & 0.1298 & 0.1572 & 0.1849 \\
\hline 0.90 & 0.90 & 0.0907 & 0.0943 & 0.1006 & 0.1102 & 0.1224 & 0.1352 \\
\hline 0.95 & 0.00 & 0.0380 & 0.0508 & 0.0781 & 0.1266 & 0.1879 & 0.2440 \\
\hline 0.95 & 0.10 & 0.0375 & 0.0492 & 0.0738 & 0.1181 & 0.1754 & 0.2289 \\
\hline 0.95 & 0.25 & 0.0370 & 0.0468 & 0.0675 & 0.1052 & 0.1559 & 0.2050 \\
\hline 0.95 & 0.50 & 0.0367 & 0.0435 & 0.0577 & 0.0838 & 0.1214 & 0.1608 \\
\hline 0.95 & 0.75 & 0.0380 & 0.0418 & 0.0494 & 0.0633 & 0.0845 & 0.1094 \\
\hline 0.95 & 0.90 & 0.0416 & 0.0432 & 0.0466 & 0.0528 & 0.0623 & 0.0741 \\
\hline
\end{tabular}


TABLE IV

Values of $G_{d}=\tau_{d} / \tau_{e}$, for various total extinction optical depths $\tau_{e 0}$, and illumination case $b$

\begin{tabular}{|c|c|c|c|c|c|c|c|}
\hline \multirow[t]{2}{*}{$\gamma$} & \multirow[t]{2}{*}{$g$} & \multicolumn{6}{|l|}{$G_{d}$} \\
\hline & & $\tau_{e 0}=1$ & $\tau_{e 0}=2$ & $\tau_{e 0}=4$ & $\tau_{e 0}=8$ & $\tau_{e 0}=16$ & $\tau_{e 0}=32$ \\
\hline 0.10 & 0.00 & 0.8577 & 0.9046 & 0.9390 & 0.9620 & 0.9767 & 0.9859 \\
\hline 0.10 & 0.10 & 0.8616 & 0.9039 & 0.9350 & 0.9557 & 0.9690 & 0.9773 \\
\hline 0.10 & 0.25 & 0.8676 & 0.9030 & 0.9290 & 0.9464 & 0.9575 & 0.9644 \\
\hline 0.10 & 0.50 & 0.8779 & 0.9017 & 0.9192 & 0.9308 & 0.9383 & 0.9430 \\
\hline 0.10 & 0.75 & 0.8887 & 0.9007 & 0.9085 & 0.9154 & 0.9192 & 0.9215 \\
\hline 0.10 & 0.90 & 0.8953 & 0.9003 & 0.9038 & 0.9061 & 0.9077 & 0.9086 \\
\hline 0.30 & 0.00 & 0.5439 & 0.6874 & 0.7958 & 0.8690 & 0.9164 & 0.9468 \\
\hline 0.30 & 0.10 & 0.5564 & 0.6864 & 0.7850 & 0.8517 & 0.8949 & 0.9225 \\
\hline 0.30 & 0.25 & 0.5762 & 0.6858 & 0.7692 & 0.8258 & 0.8624 & 0.8858 \\
\hline 0.30 & 0.50 & 0.6125 & 0.6871 & 0.7441 & 0.7831 & 0.8083 & 0.8243 \\
\hline 0.30 & 0.75 & 0.6537 & 0.6917 & 0.7209 & 0.7411 & 0.7541 & 0.7623 \\
\hline 0.30 & 0.90 & 0.6808 & 0.6962 & 0.7081 & 0.7163 & 0.7216 & 0.7249 \\
\hline 0.50 & 0.00 & 0.1809 & 0.4225 & 0.6116 & 0.7416 & 0.8257 & 0.8793 \\
\hline 0.50 & 0.10 & 0.2029 & 0.4232 & 0.5965 & 0.7161 & 0.7937 & 0.8434 \\
\hline 0.50 & 0.25 & 0.2392 & 0.4264 & 0.5747 & 0.6780 & 0.7453 & 0.7885 \\
\hline 0.50 & 0.50 & 0.3095 & 0.4387 & 0.5424 & 0.6156 & 0.6635 & 0.6943 \\
\hline 0.50 & 0.75 & 0.3946 & 0.4618 & 0.5165 & 0.5556 & 0.5813 & 0.5978 \\
\hline 0.50 & 0.90 & 0.4551 & 0.4825 & 0.5052 & 0.5215 & 0.5323 & 0.5392 \\
\hline 0.70 & 0.00 & -0.2503 & 0.0831 & 0.3568 & 0.5501 & 0.6723 & 0.7446 \\
\hline 0.70 & 0.10 & -0.2171 & 0.0882 & 0.3411 & 0.5216 & 0.6369 & 0.7066 \\
\hline 0.70 & 0.25 & -0.1615 & 0.1003 & 0.3196 & 0.4789 & 0.5823 & 0.6464 \\
\hline 0.70 & 0.50 & -0.0484 & 0.1349 & 0.2921 & 0.4097 & 0.4880 & 0.5381 \\
\hline 0.70 & 0.75 & 0.0973 & 0.1955 & 0.2809 & 0.3468 & 0.3918 & 0.4210 \\
\hline 0.70 & 0.90 & 0.2097 & 0.2511 & 0.2872 & 0.3157 & 0.3355 & 0.3484 \\
\hline 0.90 & 0.00 & -0.7825 & -0.3920 & -0.0554 & 0.1989 & 0.3563 & 0.4390 \\
\hline 0.90 & 0.10 & -0.7363 & -0.3766 & -0.0631 & 0.1783 & 0.3313 & 0.4135 \\
\hline 0.90 & 0.25 & -0.6579 & -0.3467 & -0.0710 & 0.1477 & 0.2918 & 0.3723 \\
\hline 0.90 & 0.50 & -0.4955 & -0.2726 & -0.0696 & 0.1000 & 0.2205 & 0.2937 \\
\hline 0.90 & 0.75 & -0.2716 & -0.1480 & -0.0332 & 0.0679 & 0.1464 & 0.1989 \\
\hline 0.90 & 0.90 & -0.0803 & -0.0257 & 0.0251 & 0.0713 & 0.1093 & 0.1366 \\
\hline 0.95 & 0.00 & -0.9379 & -0.5481 & -0.2159 & 0.0400 & 0.2038 & 0.2903 \\
\hline 0.95 & 0.10 & -0.8879 & -0.5283 & -0.2180 & 0.0257 & 0.1857 & 0.2718 \\
\hline 0.95 & 0.25 & -0.8033 & -0.4914 & -0.2174 & 0.0048 & 0.1571 & 0.2420 \\
\hline 0.95 & 0.50 & -0.6279 & -0.4032 & -0.1997 & -0.0252 & 0.1052 & 0.1849 \\
\hline 0.95 & 0.75 & -0.3834 & -0.2582 & -0.1414 & -0.0355 & 0.0524 & 0.1144 \\
\hline 0.95 & 0.90 & -0.1697 & -0.1137 & -0.0606 & -0.0110 & 0.0331 & 0.0678 \\
\hline
\end{tabular}


We shall call $\tau_{d}$ the exclusion optical depth, and $G_{d}$ the exclusion factor. A small value of $G_{d}\left(G_{d} \ll 1\right)$ implies that the grains exclude radiation from the cloud interior much less efficiently than their extinction optical depth $\tau_{e}$ would suggest. Conversely, if $G_{d}$ approximates to unity $\left(G_{d} \sim 1\right)$, then the extinction is mainly due to absorption, and the extinction optical depth $\tau_{e}$ is a good measure of the ability of the grains to exclude radiation from the cloud interior.

We have estimated $\tau_{d 0}$ and $G_{d}$ on the grid of $\left(\gamma, g, \tau_{e 0}\right)$ values by matching the central normalized radiation density $u_{c}$ - as derived above using the accurate numerical Equations (13a) through (16) and presented in Tables I and II - to the value given by the approximate Equations $(17 \mathrm{a}, \mathrm{b})$. When this procedure is used to establish the values of $\tau_{d 0}$ and $G_{d}$, the greatest divergence between the accurate numerical solutions (Equations (13a) through (16)) and the approximate solutions (Equations $(17 \mathrm{a}, \mathrm{b})$ ) occurs at the cloud boundary. In the worst case, this divergence may amount to a factor of two, but in general it is much smaller. Clearly the error might be reduced somewhat by matching the solutions at some intermediate optical depth; or alternatively by a least squares fitting procedure. This was deemed unnecessary, in the sense that the central radiation density is probably the most relevant quantity. For in

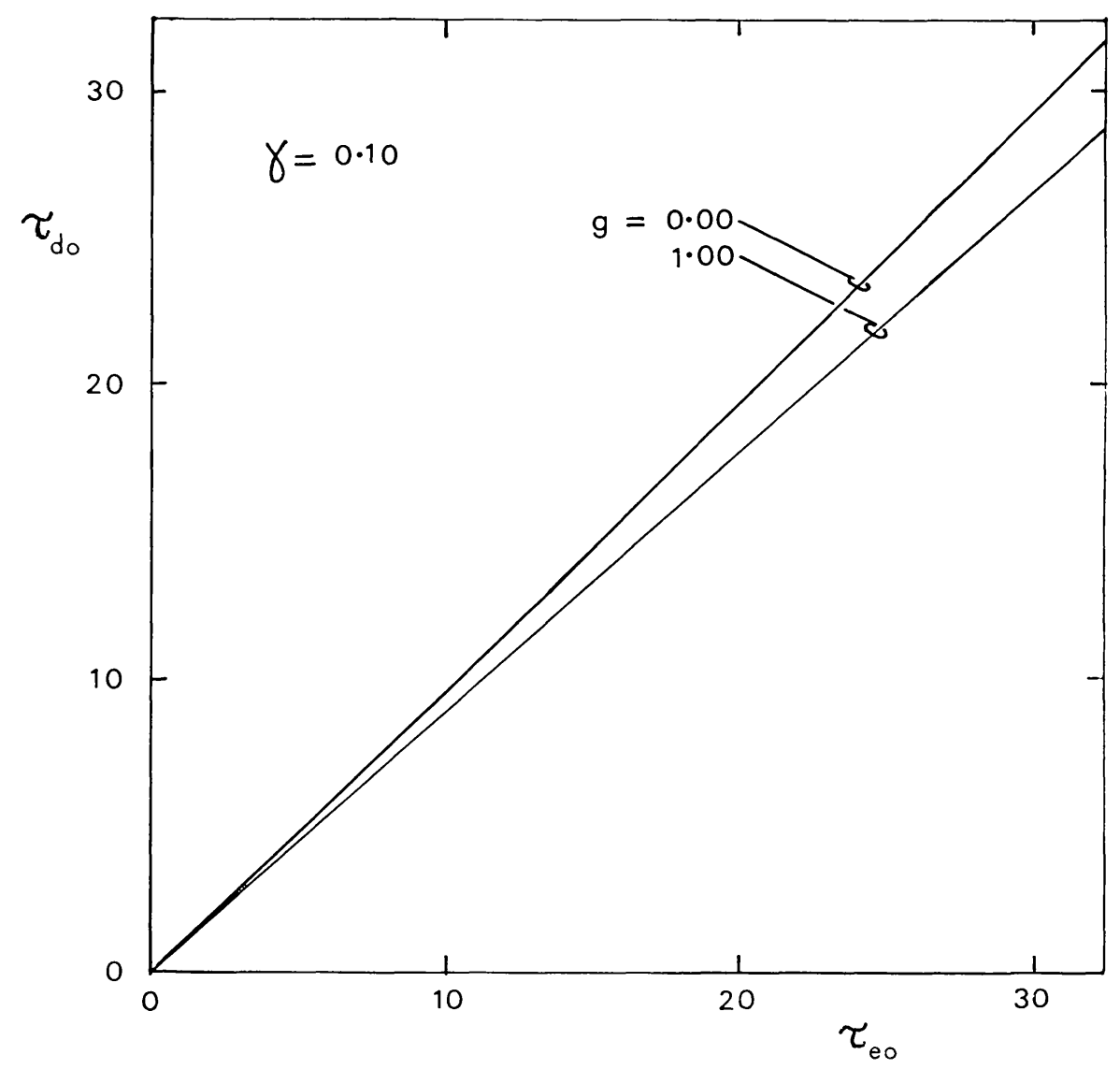

Fig. 1. The variation of $\tau_{d 0}$ with $\tau_{e 0}$ for both illumination cases (a and b); $\gamma=0.10$; and $g=0.00$, 1.00 ; the results are effectively identical for the two different illumination cases. 


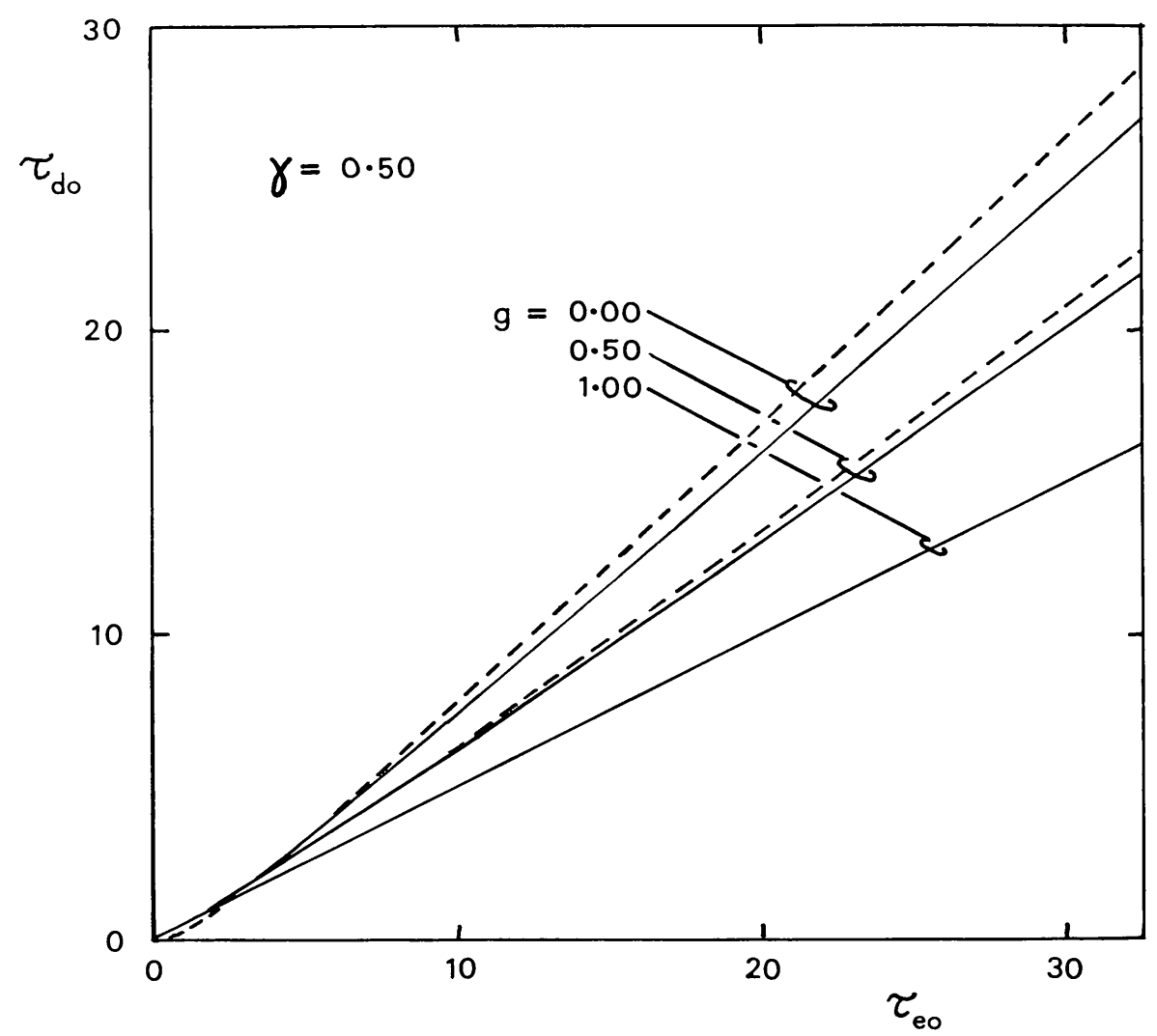

Fig. 2a. As Figure 1, but for $\gamma=0.50$; and $g=0.00,0.50,1.00$ : the full curves correspond to illumination case $a$, and the dashed curves to illumination case $b$.

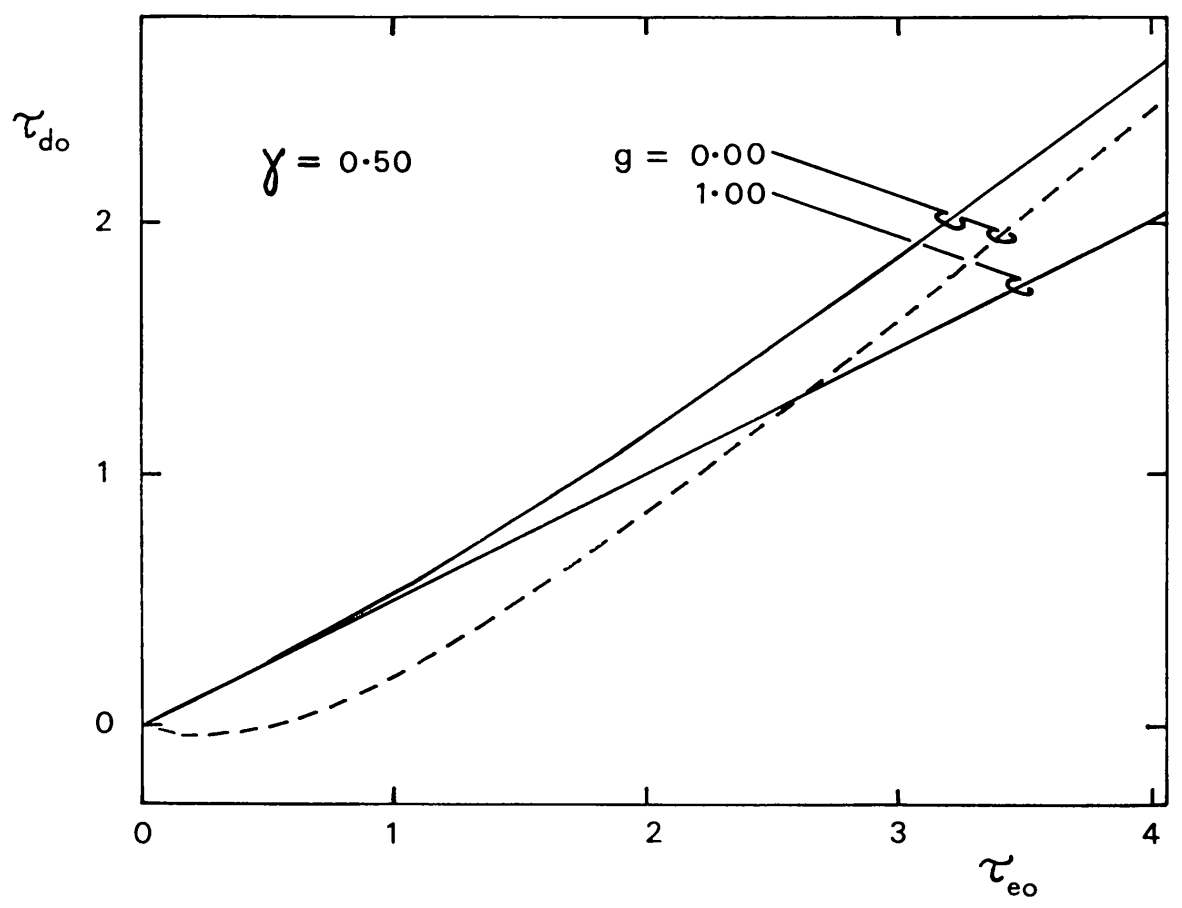

Fig. 2b. An enlargement of the part of Figure 2a near the origin. 
regions of star formation, the evolution is most strongly influenced by the conditions in the dark, dense cores of protostellar or protocluster fragments.

Table III gives values of $G_{d}$ the exclusion factor, on a grid of $\left(\gamma, g, \tau_{e 0}\right)$ values for illumination case a. The first and second columns give $\gamma$ and $g$. The remaining six columns contain the values of $G_{d}$, each column being headed by the appropriate value of $\tau_{e 0}$. Table IV is the same as Table III, but for illumination case $b$. The negative values of $G_{d}$ in Table IV correspond to negative values of $\tau_{d 0}$, which in turn correspond to the values of $u_{c}$ exceeding unity which we discussed above.

In Figures 1 through $3 \mathrm{~b}$, we have plotted $\tau_{d 0}$ against $\tau_{e 0}$. In all these figures, solid curves correspond to illumination case $a$ : the results for illumination case $b$ are marked by dashed curves, only where they differ appreciably from those for illumination case a. Figure 1 shows the results for small albedo: $(\gamma, g)=(0.10,0.00)$ and $(0.10,1.00)$. For small albedo, the exclusion optical depth $\tau_{d 0}$ is effectively independent of the illumination geometry, and varies approximately linearly with extinction

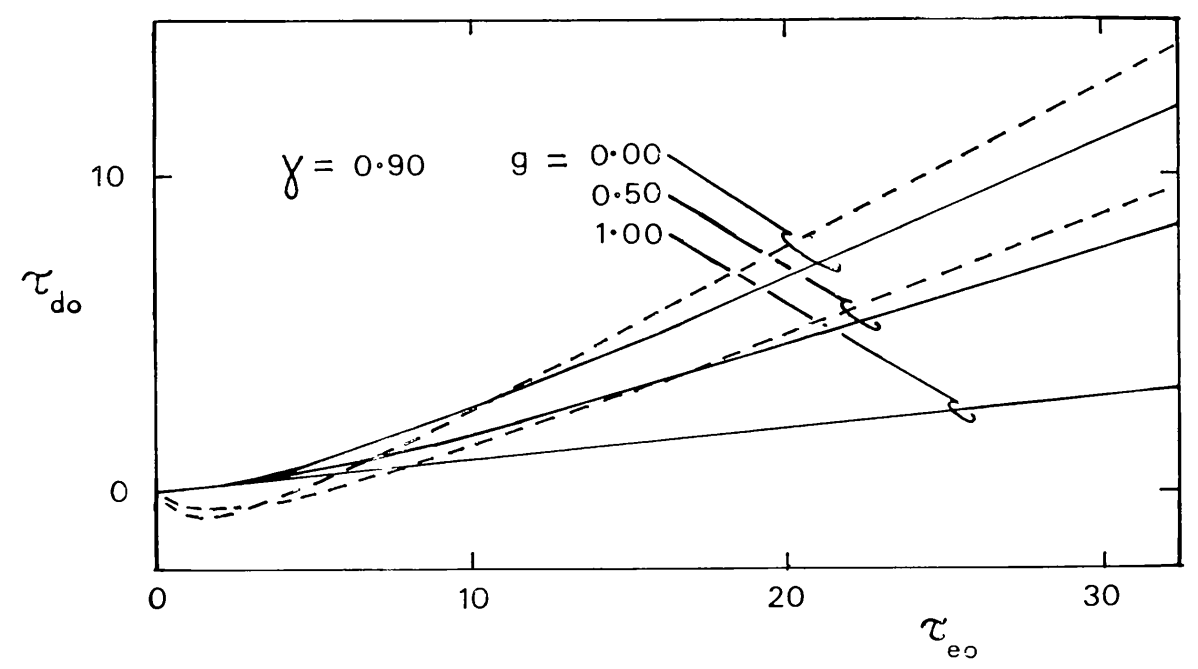

Fig. 3a. As Figure 2a, but for $\gamma=0.90$.

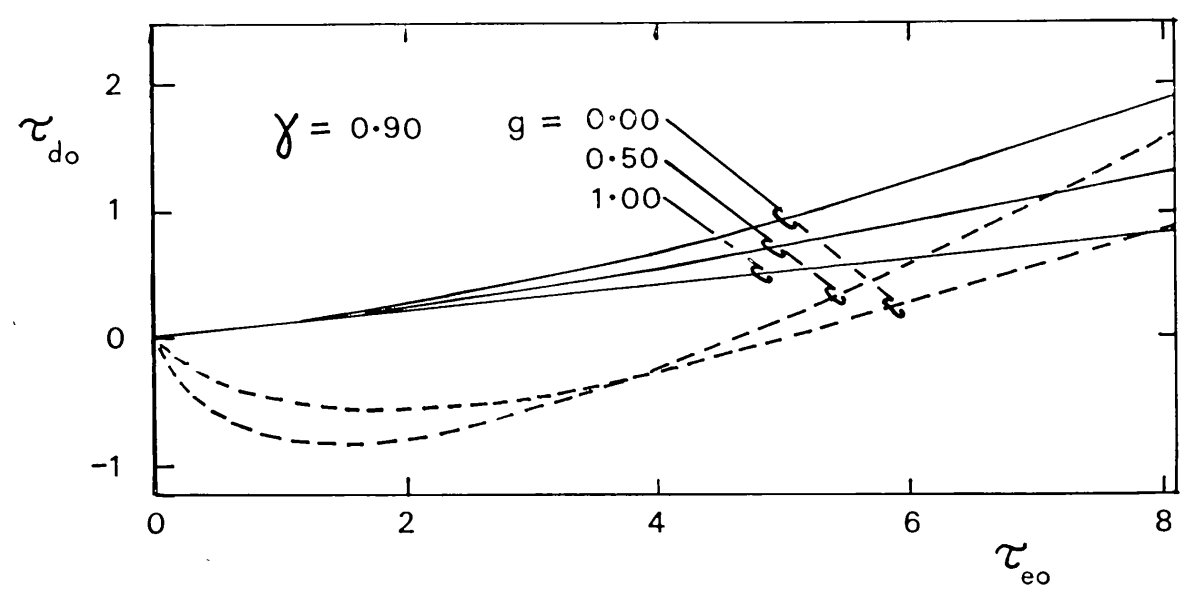

Fig. 3b. An enlargement of the part of Figure 3a near the origin. 
optical depth $\tau_{e 0}$. Figure $2 \mathrm{a}$ shows the results for intermediate albedo: $(\gamma, g)=$ $(0.50,0.00),(0.50,0.50)$ and $(0.50,1.00)$. Figure $2 \mathrm{~b}$ is an enlargement of Figure $2 \mathrm{a}$ near the origin. Figures $3 a, b$ are the same as Figures $2 a, b$, except that here the albedo is large, $\gamma=0.90$. We see that as $\gamma$ is increased, the relationship between $\tau_{d 0}$ and $\tau_{e 0}$ comes to depend increasingly on the illumination geometry; is increasingly non-linear; and admits increasingly larger negative $\tau_{d 0}$ values for illumination case $b$ (corresponding to amplification of the radiation field in an anisotropically illuminated cloud).

Given the forms of the curves illustrated in Figures 1 through $3 \mathrm{~b}$, and the spacing of the tabulated points, it is clear that $G_{d}$ values can easily be obtained for arbitrary $\left(\gamma, g, \tau_{e 0}\right)$ by simple extrapolation using the grid of values in Tables III and IV. Equation (18) can then be used to evaluate the exclusion optical depth $\tau_{d 0}$.

\section{Concluding Remarks}

We submit that the concept of an exclusion optical depth is meaningful and numerically convenient in evaluating the role of UV radiation in dark clouds. With simple exponential integral or exponential forms for the attenuation of the radiation energy density (i.e. Equations $(17 \mathrm{a}, \mathrm{b})$ ), a phenomenon involving these radiations can be clearly formulated, without becoming lost in complicated auxiliary equations of radiative transfer. There are many phenomena involving UV radiations which may be important in the interiors of dark clouds. Firstly the radiations may be powerful heating agents (primarily for the grains). Secondly, they may be powerful ionizing agents, or rather more generally, agents of disruption.

UV and visual radiations are the main heating agents for grains, and thus play a dominant role in determining grain temperatures. Even in the dense dark regions where UV radiations do not penetrate, their influence is still felt by the grains, in the sense that the thermal IR radiation which does penetrate is mainly excited by UV radiations in less well shielded parts of the cloud. By influencing grain temperatures - either directly or indirectly - UV radiations can also strongly influence the state of the gas: firstly, because at sufficiently high densities and/or large optical depths, the gas and radiation field tend to thermal equilibrium with the grains; and secondly, because rates for molecule formation by surface recombination on grains are strongly dependent on the grain temperature (e.g. Hollenbach and Salpeter, 1970).

By interacting with a grain, an individual photon may cause the ejection of an electron, thus giving the grain a positive charge (Watson, 1972); or it may remove a newly formed molecule from the grain surface (Watson and Salpeter, 1972a). By interacting with a grain, a UV photon may also alter the IR radiative properties of the grain, and hence affect its thermal balance (Hoyle and Wickramasinghe, 1967); and it may affect the suitability of the surface for molecule formation by creating sites of peculiar binding (Hollenbach and Salpeter, 1970). In interacting with the gas, 


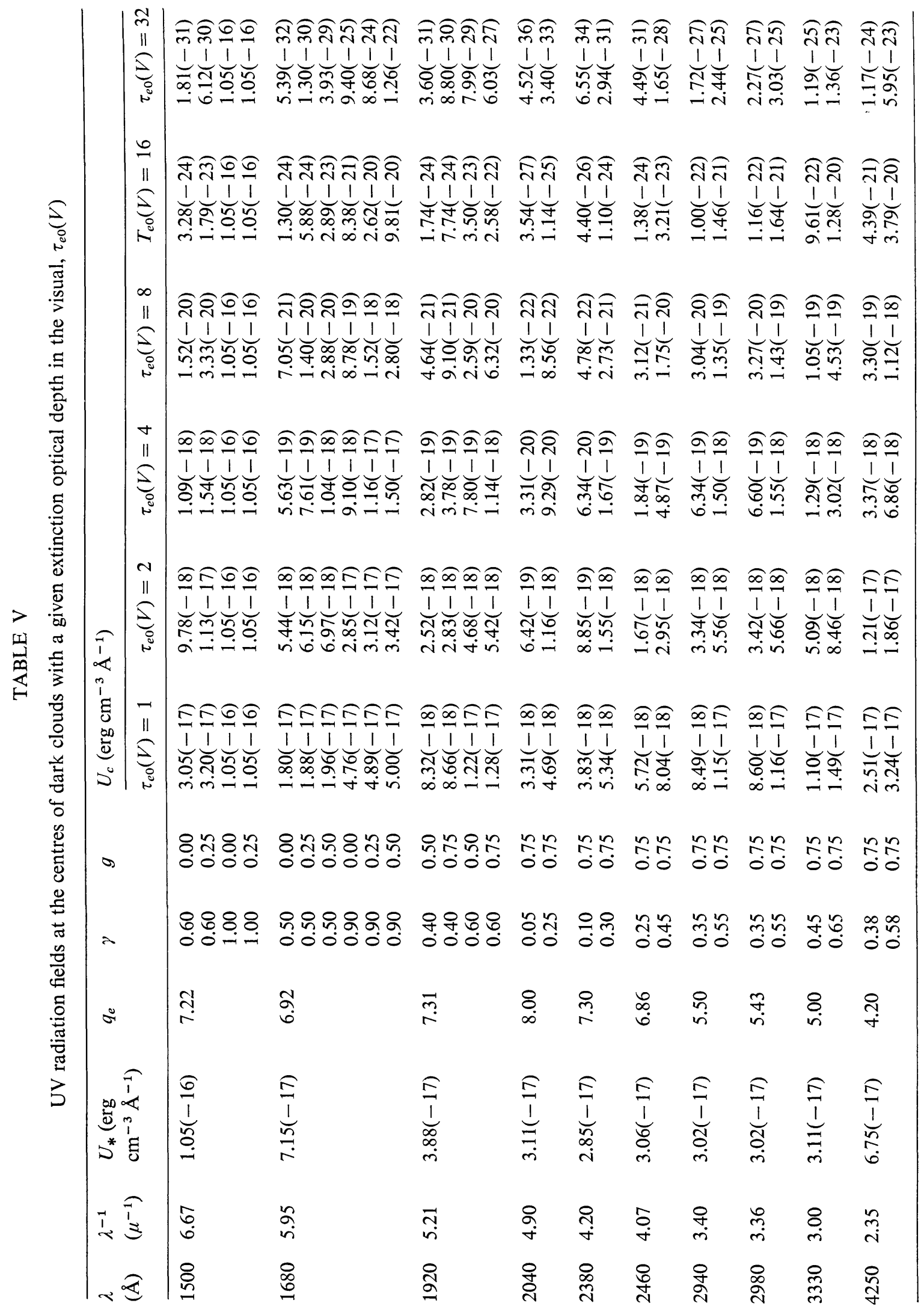


an individual photon may cause ionization, thereby increasing the electron density, and influencing the general ionization balance (e.g. Brown, 1972). An ion formed in this way may play an important role in the chemistry of the gas, either by undergoing a two-body reaction (in the gas phase) to form a molecule, or - if it is a molecular ion - by recombining dissociatively (Solomon and Klemperer, 1972; Watson and Salpeter, 1972b; cf. Herbst and Klemperer, 1973). By increasing the density of charged particles, UV radiation may also influence the dynamic evolution of the medium, by enhancing the frictional coupling between gas particles and magnetic flux (Mestel and Spitzer, 1956). (Either electrons or charged grains may be effective in this respect.) Finally, the grain charge can affect the growth of the grain itself, and hence the efficiency of depletion by accretion: it may also influence the alignment of the grain.

Evidently, it is important to know the UV radiation field within a dark cloud. To this end, we have taken the data of Witt and Johnson (1973) on the radiation energy density in the solar vicinity (assumed to be representative of the general interstellar medium), and the data of Witt and Lillie (1973) on the scattering properties of interstellar dust grains; and we have used them to compute the energy density in (1200, 4500) $\AA$ at the centre of a dark cloud. Witt and Lillie (1973) give errors for the albedo, and we have therefore done the computations for a maximum albedo, and for a minimum albedo. Similarly, at short wavelengths, Witt and Lillie (1973) give only an approximate upper limit to $g$, and we have therefore treated a range of $g$ values consistent with this limit. We have used the illumination geometry of case a (diffuse illumination), and have labelled the clouds according to their total extinction optical depth in the visual $\tau_{e 0}(V)$. The results are presented in Table $V$ and Figures $4 \mathrm{a}, \mathrm{b}, \mathrm{c}$, $\mathrm{d}$, e. In Table $\mathrm{V}$, the first and second columns give the wavelength $\lambda$ in ångstroms, and the inverse wavelength $\lambda^{-1}$ in inverse microns; the third column gives the unshielded radiation density $U_{*}$ (Witt and Johnson, 1973) and this is plotted in Figure $4 \mathrm{a}$; the fourth column gives the extinction efficiency $q_{e}$ (Bless and Savage, 1972); the fifth and sixth columns give the albedo $\gamma$, and the mean scattering-angle cosine $g$ (Witt and Lillie, 1973). The remaining six columns give the central radiation density $u_{c}$, each column being headed by the appropriate value of the total extinction optical depth in the visual $\tau_{e 0}(V)$. In Figures $4 \mathrm{~b}, \mathrm{c}, \mathrm{d}$, e we have plotted the results for $\tau_{e 0}(V)=4,8,16,32$. In each case we give the maximum and the minimum radiation field allowed by the uncertainties in the albedo and the mean scattering-angle cosine: the area in between the maximum and the minimum is shaded. We see that in the interior of a dark cloud $\left(\tau_{e 0}(V) \gtrsim 4\right)$, the radiation field around the $2200 \AA$ ' hump' is very weak, since the extinction here derives mainly from absorption. However, at shorter and longer wavelengths, the radiation field is much less strongly attenuated. This is because at shorter wavelengths $(\lambda<2200 \AA)$ the albedo is very high $(\gamma \rightarrow 1$, i.e. the extinction derives mainly from scattering), but the scattering phase-function $P_{s}(\theta)$ is only weakly forward-throwing, if at all. At longer wavelengths $(\lambda>2200 \AA)$, the albedo is moderately high $(\gamma \sim 0.5$, i.e. the extinction is approximately half 


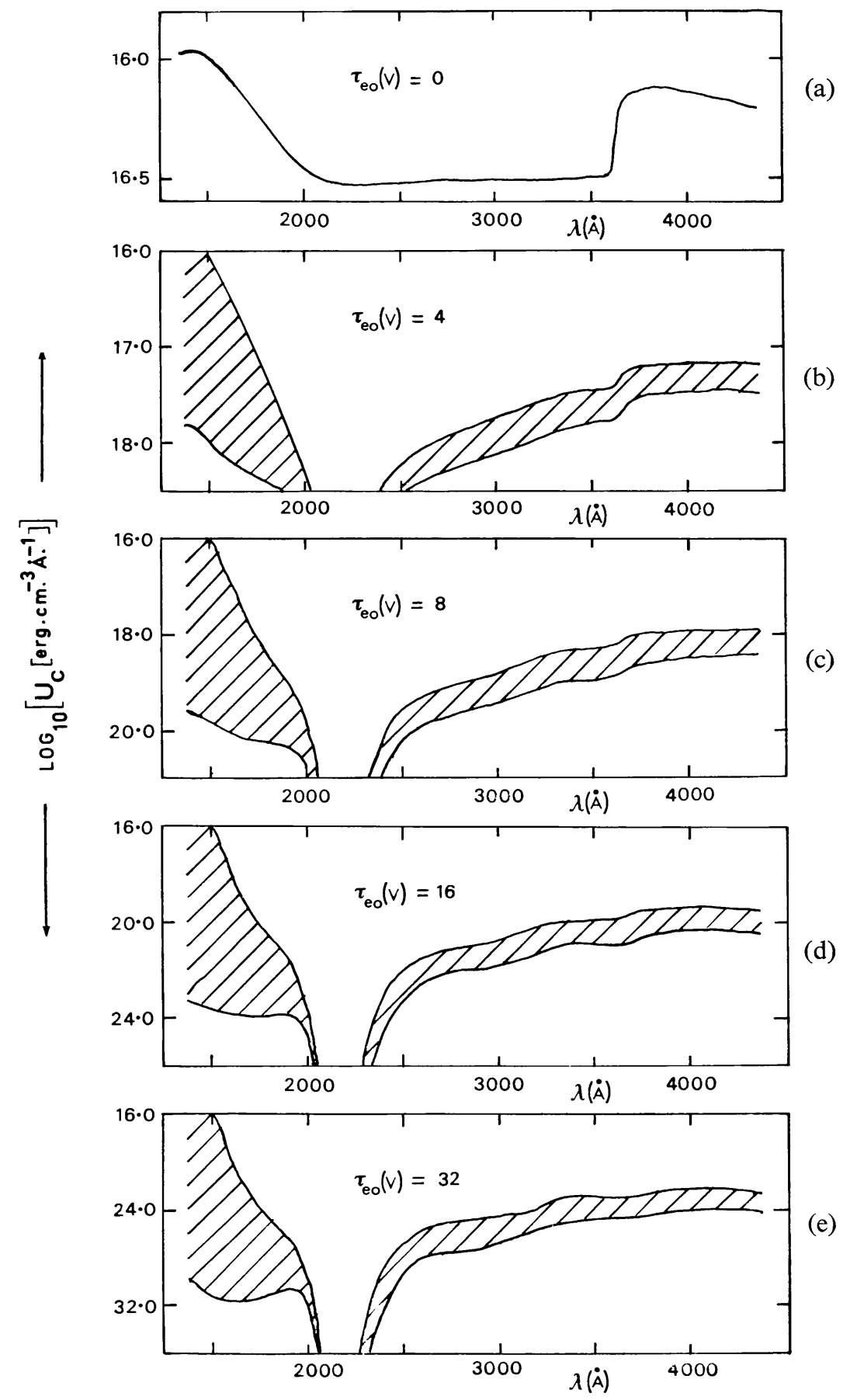

Fig. 4a. The unobscured radiation density $U_{*}\left(\mathrm{erg} \mathrm{cm}^{-3} \AA^{-1}\right)$ in the solar vicinity (from Witt and Lillie, 1973).

Fig. 4b. The radiation density $U_{c}\left(\mathrm{erg} \mathrm{cm}^{-3} \AA^{-1}\right)$ at the centre of a dark cloud having total extinction optical depth in the visual $\tau_{e 0}(V)=4$. The upper and lower curves bounding the shaded area are the maximum and minimum densities allowed by the uncertainties in the grain properties.

Fig. 4c. As Figure 4b, but for $\tau_{e 0}(V)=8$.

Fig. 4d. As Figure $4 \mathrm{~b}$, but for $\tau_{e 0}(V)=16$.

Fig. 4e. As Figure $4 \mathrm{~b}$, but for $\tau_{e 0}(V)=32$. 
absorption and half scattering), and the scattering phase-function $P_{s}(\theta)$ is strongly forward-throwing $(g \sim 0.75)$. Thus, with the exception of a narrow band around $2200 \AA$, UV radiation apparently penetrates rather efficiently into dark clouds, and must therefore play a number of important roles in the physics of the interiors of such clouds, as enumerated above. Whilst it is beyond the scope of this paper to evaluate these roles, we should note that the short wavelength photons $(\lambda<2200 \AA)$ are the most energetic, and thus in general potentially the most influential. It is therefore unfortunate (although not surprising) that the results are most uncertain for these short wavelengths, and it is to be hoped that these uncertainties can be reduced in the near future. We should also note that most of the important phenomena involving UV photons which may take place in the interiors of dark clouds are not resonant. Thus they will be significant even if UV radiation penetrates the cloud efficiently only over a rather narrow band of wavelengths: the interacting species are, in the main, not very particular about the exact wavelength of the photon they receive, provided that this photon has sufficient energy.

\section{References}

Aannestad, P. A. and Purcell, E. M.: 1973, Ann. Rev. Astron. Astrophys. 11, 309.

Abramowitz, M. and Stegun, I. A.: 1964, Handbook of Mathematical Functions, Dover, New York. Bless, R. C. and Savage, B. D.: 1972, Astrophys. J. 171, 293.

Brown, R. L.: 1972, Astrophys. J. 173, 593.

Carrasco, L., Strom, S. E., and Strom, K. M.: 1973, Astrophys. J. 182, 95.

Herbst, E. and Klemperer, W.: 1973, Astrophys. J. 185, 505.

Hollenbach, D. and Salpeter, E. E.: 1970, J. Chem. Phys. 53, 79.

Hoyle, F. and Wickramasinghe, N. C.: 1967, Nature 214, 969.

Mestel, L. and Spitzer, L., Jr.: 1956, Monthly Notices Roy. Astron. Soc. 116, 503.

Solomon, P. M. and Klemperer, W.: 1972, Astrophys. J. 178, 389.

Watson, W. D.: 1972, Astrophys. J. 176, 103.

Watson, W. D. and Salpeter, E. E.: 1972a, Astrophys. J. 174, 321.

Watson, W. D. and Salpeter, E. E.: 1972b, Astrophys. J. 175, 659.

Werner, M. W. and Salpeter, E. E.: 1969, Monthly Notices Roy. Astron. Soc. 145, 249.

Whitworth, A. P.: 1972, unpublished Ph.D. dissertation, Manchester.

Wickramasinghe, N. C.: 1973, Light Scattering Functions for Small Particles, Hilger, London.

Wickramasinghe, N. C. and Nandy, K.: 1972, Rep. Prog. Phys. 35, 157.

Witt, A. N. and Johnson, M. W.: 1973, Astrophys. J. 181, 363.

Witt, A. N. and Lillie, C. F.: 1973, Astron. Astrophys. 25, 397. 